\title{
Emergent Spacetime
}

\author{
Nathan Seiberg \\ School of Natural Sciences, \\ Institute for Advanced Study, \\ Princeton, NJ 08540 USA \\ seiberg@ias.edu
}

\begin{abstract}
We summarize the arguments that space and time are likely to be emergent notions; i.e. they are not present in the fundamental formulation of the theory, but appear as approximate macroscopic concepts. Along the way we briefly review certain topics. These include ambiguities in the geometry and the topology of space which arise from dualities, questions associated with locality, various known examples of emergent space, and the puzzles and the prospects of emergent time.
\end{abstract}

Rapporteur talk at the 23rd Solvay Conference in Physics, December, 2005. 


\section{Introduction}

The purpose of this talk is to review the case for the idea that space and time will end up being emergent concepts; i.e. they will not be present in the fundamental formulation of the theory and will appear as approximate semiclassical notions in the macroscopic world. This point of view is widely held in the string community and many of the points which we will stress are well known.

Before we motivate the idea that spacetime should be emergent, we should discuss the nature of space in string theory. We do that in section 2, where we review some of the ambiguities in the underlying geometry and topology. These follow from the dualities of string theory. T-duality leads to ambiguities at the string length $l_{s}$ and the quantum dualities lead to ambiguities at the Planck length $l_{p} \ll l_{s}$. All these ambiguities in the geometry are associated with the fact that as we try to probe the space with increasing resolution, the probes we use become big and prevent us from achieving the desired accuracy.

The discussion about ambiguities in space will lead us to make some comments about locality. In particular, we will ask whether to expect locality in a space or in one of its duals.

In section 3 we will briefly mention some of the peculiar non-gravitational theories which are found as certain limits of string theory. Some of them are expected to be standard field theories, albeit without a Lagrangian. Others, like theories on a noncommutative space or little string theory, are not local quantum field theory. They exhibit interesting nonlocal behavior.

In section 4 we will make the case that general covariance is likely to be a derived concept.

Section 5 will present several examples of emergent space. First we will discuss the simplest examples which do not involve gravity. Then we will turn to four classes of examples of emergent space: the emergent two-dimensional (worldsheet) gravity from the matrix model, the celebrated gauge/gravity 
duality, linear dilaton backgrounds, and the BFSS matrix model. We will discuss some of their properties and will stress the similarities and the differences between them. In particular, we will discuss their finite temperature behavior as a diagnostic of the system in extreme conditions.

Section 6 will be devoted to emergent time. Here we do not have concrete examples. Instead, we will present some of the challenges and confusions that this idea poses. We will also mention that understanding how time emerges will undoubtedly shed new light on some of the most important questions in theoretical physics including the origin of the Universe.

We will summarize the talk in section 7 where we will also present some general speculations.

Before we start we should mention some important disclaimers. As we said, most of the points which will be discussed here are elementary and are well known in the string community. We apologize for boring you with them. Other points will be inconclusive because they reflect our confusions. Also, not all issues and all points of view will be presented. Instead, the presentation will be biased by my prejudice and my own work. For example, the discussion will focus on string theory (for textbooks, see [1, 2]), and other approaches to quantum gravity will not be reviewed. Since this talk is expected to lead to a discussion, we will present certain provocative and perhaps outrageous ideas. Finally, there will be very few references, mostly to reviews of the subject, rather than to original papers.

\section{Ambiguous space}

\subsection{Ambiguous space in classical string theory}

We start this section by discussing the ambiguities in the geometry and the topology which exist already at string tree level. These are usually referred to as T-duality (for reviews, see e.g. [3, 4]).

Consider strings propagating in some background fields (e.g. metric). 
Clearly, these background fields should satisfy the equations of motion. Then, it turns out that different backgrounds can lead to the same physics without any observable difference between them. Therefore, there is no unique answer to the question: "What is the background metric?" and the background geometry is ambiguous.

Intuitively, these ambiguities arise from the extended nature of the string. Features in the geometry which are smaller than the string length $l_{s}=\sqrt{\alpha^{\prime}}$ cannot be detected using a string probe whose characteristic size is $l_{s}{ }^{1}{ }^{1}$

The simplest and most widely known example of this ambiguity is the equivalence between a circle with radius $R$ and a circle with radius $\alpha^{\prime} / R$. A slightly more peculiar example is the equivalence between a circle with radius $R=2 \sqrt{\alpha^{\prime}}$ and a $\mathcal{Z}_{2}$ quotient of a circle (a line segment) with $R=\sqrt{\alpha^{\prime}}$. This example demonstrates that even the topology is ambiguous. Furthermore, we can start with a circle of radius $R$, smoothly change it to $R=2 \sqrt{\alpha^{\prime}}$, then use the duality with the line segment and then change the length of the line segment. This way we start with a circle which is not dual to a line segment and we continuously change its topology to a line segment which is not dual to a circle.

A characteristic feature of these dualities is the role played by momentum and winding symmetries. In the example of the two circles with radii $R$ and $\alpha^{\prime} / R$ momentum conservation in one system is mapped to winding conservation in the other. Momentum conservation arises from a geometric symmetry (an isometry) of the circle. It is mapped to winding conservation which is a stringy symmetry. This is a manifestation of the stringy nature of T-duality and it makes it clear that it is associated with the extended nature of the string.

In some situations there exists a description of the system in terms of a macroscopic background; i.e. the space and all its features are larger than

\footnotetext{
${ }^{1}$ D-branes [2] which are smaller than $l_{s}$ can sometime lead to a more precise metric, but different kinds of D-branes lead to different answers and therefore the ambiguity is not resolved.
} 
$l_{s}$. This is the most natural description among all possible dual descriptions. However, two points should be stressed about this case. First, even though this description is the most natural one, there is nothing wrong with all other T-dual descriptions and they are equally valid. Second, it is never the case that there is more than one such macroscopic and natural description.

More elaborate and richer examples of this fundamental phenomenon arise in the study of Calabi-Yau spaces. Here two different Calabi-Yau spaces which are a "mirror pair" (for a review, see e.g. [5]) lead to the same physics. Furthermore, it is often the case that one can continuously interpolate between different Calabi-Yau spaces with different topology. These developments had dramatic impact on mathematics (see e.g. [5, 6]).

Another kind of T-duality is the cigar/Sine-Liouville duality [7]. One side of the duality involves the cigar geometry: a semi-infinite cylinder which is capped at one side. It has a varying dilaton, such that the string coupling at the open end of the cigar vanishes. This description makes it clear that the shift symmetry around the cigar leads to conserved momentum. However, the string winding number is not conserved, because wound strings can slip through the capped end of the cigar. The other side of this duality involves an infinite cylinder. Here the winding conservation is broken by a condensate of wound strings. The cigar geometry is described by a two-dimensional field theory with a nontrivial metric but no potential, while its dual, the SineLiouville theory, is a theory with a flat metric but a nontrivial potential. This example again highlights the importance of the winding modes. It also demonstrates that the T-duality ambiguity is not limited to compact dimensions. Here the ambiguity is between two different non-compact systems (an infinite and a half infinite cylinder).

From the worldsheet point of view T-duality represents an exact equivalence between different two-dimensional conformal field theories. Therefore, the phenomenon of T-duality persists beyond classical string theory, and extends to all orders in perturbation theory. Furthermore, in some situations 
one can argue that T-duality is a gauge symmetry. This observation means that T-duality is exact and it cannot be violated non-perturbatively.

The phenomenon of T-duality leads us to ask two interesting questions. First, is $l_{s}$ a minimum length; i.e. is the notion of distance ill defined below $l_{s}$ ? Second, is the theory local in one space, or in its T-dual space, or in neither? We will return to these questions below.

Before we leave the topic of ambiguities in classical string theory we would like to mention another important stringy phenomenon which is associated with the extended nature of the string. The high energy density of string states is such that the canonical ensemble of free strings does not exist above a certain temperature $T_{H} \sim \frac{1}{l_{s}}$, which is known as the Hagedorn temperature [1, 2. The relevant modes which lead to this phenomenon are long strings. They have large entropy and hence the partition function diverges at $T_{H}$. Equivalently, when Euclidean time is compactified on a circle of radius $R=$

$\frac{1}{2 \pi T}$ (with thermal boundary conditions) an instability appears when $R \leq$

$\frac{1}{2 \pi T_{H}}$. This instability is associated with strings which are wound around the Euclidean time circle. $T_{H}$ could be a limiting temperature, beyond which the theory does not exist. Alternatively, this phenomenon could mean that the system undergoes a first order phase transition to another phase. That phase could exhibit the fundamental degrees of freedom more clearly. Again we see that the theory tries to hide its short distance behavior.

\subsection{Ambiguous space in quantum string theory}

Quantum mechanics introduces new ambiguities in space which are related to new dualities (for reviews, see e.g. [2, 4]). These ambiguities go beyond the obvious ambiguities due to the quantum fluctuations. Here the characteristic length scale is the Planck length $l_{p} \ll l_{s}$.

An intuitive argument explaining the origin of these ambiguities is the following. If we want to explore space with resolution of order $r$, the uncertainly principle tells us that we need to use energy $E>\frac{1}{r}$. This energy has 
to be concentrated in a region of size $r$. But in the presence of gravitational interactions, this concentration of energy creates a black hole unless $r>l_{p}$. Therefore, we cannot explore distances smaller than the Planck length.

It is important to stress that although the ambiguities in the quantum theory are often described as of different nature than the ambiguities in the classical theory, fundamentally they are quite similar. Both of them are associated with the breakdown of the standard small distance/high energy connection - as we try to increase the energy of a probe it becomes bigger and does not allow us to explore short distances.

The quantum dualities, which are also known as S-duality or U-duality, extend the classical T-duality and lead to a beautiful and coherent picture of stringy dualities. These exchange highly quantum situations with semiclassical backgrounds, exchange different branes, etc. As in the classical dualities, among all dual descriptions there is at most one description which is natural because it is semiclassical. All other dual descriptions are very quantum mechanical.

\subsection{Comments about locality}

We now turn to some comments about locality in string theory.

Quantum field theory is local. This locality guarantees that the theory is causal. We would like string theory also to be causal or at least macroscopically causal. Furthermore, we know that at long distances string theory behaves like quantum field theory and therefore it is macroscopically local. But is string theory local also over short distances?

One piece of evidence in favor of locality is the analyticity of the perturbative string S-matrix. Normally, causality and locality lead to analyticity. Since the string S-matrix is analytic, it is likely that string theory is local. However, it is logically possible that a slightly weaker condition than locality and therefore of causality can also guarantee the analyticity of the S-matrix.

One reason string theory might not be local in a standard way is the 
extended nature of the interacting objects, the strings. At the most naive and intuitive level locality of string interactions is not obvious. Even though two strings interact at a point to form a third string, this interaction is nonlocal when viewed from the point of view of the center of masses of the interacting strings. It is known that this nonlocality is harmless and is consistent with the analyticity of the S-matrix. ${ }^{2}$

We would like to comment about locality and the cosmological constant. The old fashioned point of view of the cosmological constant problem suggested that its value is related to some kind of a UV/IR mixing and to violation of naive locality - the short distance theory somehow reacts to long distance fluctuations and thus sets the value of the cosmological constant. A more modern point of view on the subject is that the cosmological constant is set anthropically (see, e.g. [8]). It remains to be seen whether the cosmological constant is a hint about some intrinsic nonlocality in the theory.

The ambiguities we discussed above might hint at some form of nonlocality. We have stressed that increasing the energy of a probe does not lead to increased resolution. Instead, the probe becomes bigger and the resolution is reduced. This point is at the heart of the various dualities and ambiguities in the background. We have already asked whether we expect locality in a space, or in its dual space. It is hard to imagine that the theory can be simultaneously local in both of them. Then, perhaps it is local in neither. Of course, when a macroscopic weakly coupled natural description exists, we expect the theory to be at least approximately local in that description.

It is important to stress that although intuitively the notion of locality is obvious, this is not the case in string theory or in any generally covariant theory. The theory has no local observables. Most of the observables are related to the S-matrix or other objects at infinity. These do not probe the detailed structure of the theory in the interior. Therefore, without local

\footnotetext{
${ }^{2}$ In open string field theory a basis based on the string midpoint replaces the basis based on the center of mass and then the interaction appears to be local.
} 
observables it is not clear how to precisely define locality.

We will argue below that space and time should be emergent concepts. So if they are not fundamental, the concept of locality cannot be fundamental as well. It is possible that locality will end up being ill defined, and there will be only an approximate notion of locality when there is an approximate notion of spacetime.

\section{Non-standard theories without gravity}

Next, let us digress slightly to review some of the non-standard theories without gravity that were found by studying various limits of string theory. These theories exhibit interesting and surprising new phenomena. We expect that these theories and their peculiar phenomena will be clues to the structure of the underlying string theory. Since they are significantly simpler than string theory, they could be used as efficient laboratories or toy models.

The first kind of surprising theories are new local field theories which cannot be given a standard Lagrangian description. These are superconformal field theories in five or six dimensions with various amount of supersymmetry. The most symmetric examples are the six-dimensional $(2,0)$ theories (for a review, see e.g. 9]). They are found by taking an appropriate scaling limit of string theory in various singularities or on coincident 5-branes. The existence of these theories calls for a new formulation of local quantum field theory without basing it on a Lagrangian.

Another class of interesting non-gravitational theories are field theories on noncommutative spaces (for a review, see e.g. [10]). These theories do not satisfy the standard rules of local quantum field theory. For example, they exhibit a UV/IR mixing which is similar to the UV/IR mixing in string theory - as the energy of an object is increased its size becomes bigger.

The most enigmatic theories which are derived from string theory are the little string theories (for a review, see e.g. [11]). These non-gravitational 
theories exhibit puzzling stringy behavior. The stringy nature of these theories arises from the fact that they appear by taking a certain scaling limit of string theory (in the presence of NS5-branes or some singularities) while keeping $\alpha^{\prime}$ fixed. One stringy phenomenon they exhibit is T-duality. This suggests that despite the lack of gravity, these theories do not have a local energy momentum tensor. Otherwise, there should have been several different energy momentum tensors which are related by T-duality. It was also argued that because of their high energy behavior these theories cannot have local observables. Finally, these theories exhibit Hagedorn spectrum with a Hagedorn temperature which is below $T_{H}$ of the underlying string theory. It was suggested that this Hagedorn temperature is a limiting temperature; i.e. the canonical ensemble does not exist beyond that temperature.

\section{Derived general covariance}

The purpose of this section is to argue that general covariance which is the starting point of General Relativity might not be fundamental. It could emerge as a useful concept at long distances without being present in the underlying formulation of the theory.

General covariance is a gauge symmetry. As with other gauge symmetries, the term "symmetry" is a misnomer. Gauge symmetries are not symmetries of the Hilbert space; the Hilbert space is invariant under the entire gauge group. Instead, gauge symmetries represent a redundancy in our description of the theory. (It is important to stress, though, that this is an extremely useful redundancy which allows us to describe the theory in simple local and Lorentz invariant terms.)

Indeed, experience from duality in field theory shows that gauge symmetries are not fundamental. It is often the case that a theory with a gauge symmetry is dual to a theory with a different gauge symmetry, or no gauge symmetry at all. A very simple example is Maxwell theory in $2+1$ dimen- 
sions. This theory has a $U(1)$ gauge symmetry, and it has a dual description in terms of a free massless scalar without a local gauge symmetry. More subtle examples in higher dimensions were found in supersymmetric theories (for reviews, see e.g. [12, 13]).

If ordinary gauge symmetries are not fundamental, it is reasonable that general covariance is also not fundamental. This suggests that the basic formulation of the theory will not have general covariance. General covariance will appear as a derived (and useful) concept at long distances.

An important constraint on the emergence of gauge symmetries follows from the Weinberg-Witten theorem [14]. It states that if the theory has massless spin one or spin two particles, these particles are gauge particles. Therefore, the currents that they couple to are not observable operators. If these gauge symmetries are not present in some formulation of the theory, these currents should not exist there. In particular, it means that if an ordinary gauge symmetry emerges, the fundamental theory should not have this symmetry as a global symmetry. In the context of emergent general covariance, this means that the fundamental theory cannot have an energy momentum tensor.

If we are looking for a fundamental theory without general covariance, it is likely that this theory should not have an underlying spacetime. This point is further motivated by the fact that General Relativity has no local observables and perhaps no local gauge invariant degrees of freedom. Therefore, there is really no need for an underlying spacetime. Spacetime and general covariance should appear as approximate concepts which are valid only macroscopically.

\section{Examples of emergent space}

\subsection{Emergent space without gravity}

The simplest examples of emergent space are those which do not involve gravity. Here the starting point is a theory without a fundamental space, 
but the resulting answers look approximately like a theory on some space. The first examples of this kind were the Eguchi-Kawai model and its various variants (for a review, see e.g. [15]). Here a $d$ dimensional $S U(N)$ gauge theory is formulated at one point. The large $N$ answers look like a gauge theory on a macroscopic space.

Certain extensions of the (twisted) Eguchi-Kawai model are theories on a noncommutative space (for a review, see e.g. [10]). Here the coordinates of the space do not commute and are well defined only when they are macroscopic.

A physical realization of these ideas is the Myers effect [16]. Here we start with a collection of $N$ branes in some background flux. These branes expand and become a single brane of higher dimension. The new dimensions of this brane are not standard dimensions. They form a so-called "fuzzy space." In the large $N$ limit the resulting space becomes macroscopic and its fuzzyness disappears.

\subsection{Emergent space with gravity: matrix model of $2 \mathrm{~d}$ gravity}

The first examples of emergent space with gravity and general covariance arose from the matrix model of random surfaces (for a review, see e.g. [17]). Here we start with a certain matrix integral or matrix quantum mechanics and study it in perturbation theory. Large Feynman diagrams of this perturbation expansion can be viewed as discretized two-dimensional surfaces.

This system is particularly interesting when the size of the matrices $N$ is taken to infinity together with a certain limit of the parameters of the matrix integral. In this double scaling limit the two-dimensional surfaces become large and smooth and the system has an effective description in terms of random surfaces. The degrees of freedom on these surfaces are local quantum fields including a dynamical metric and therefore this description is generally covariant.

The formulation of these theories as matrix models does not have a two- 
dimensional space nor does it have general covariance. These concepts emerge in the effective description.

In addition to being interesting and calculable models of two-dimensional gravity, these are concrete examples of how space and its general covariance can be emergent concepts.

\subsection{Emergent space with gravity: Gauge/Gravity du- ality}

The most widely studied examples of emergent space with gravity are based on the AdS/CFT correspondence [18, 19, 20, 21]. This celebrated correspondence is the duality between string theory in AdS space and a conformal field theory at its boundary. Since other speakers in this conference will also talk about it, we will only review it briefly and will make a few general comments about it.

The bulk theory is a theory of gravity and as such it does not have an energy momentum tensor. The dual field theory on the boundary has an energy momentum tensor. This is consistent with the discussion above about emergent gravity (section 4), because the energy momentum tensor of the field theory is in lower dimensions than the bulk theory and reflects only its boundary behavior.

The operators of the boundary theory are mapped to string states in the bulk. A particularly important example is the energy momentum tensor of the boundary theory which is mapped to the bulk graviton. The correlation functions of the conformal field theory are related through the correspondence to string amplitudes in the AdS space. (Because of the asymptotic structure of AdS, these are not S-matrix elements.) When the field theory is deformed by relevant operators, the background geometry is slightly deformed near the boundary but the deformation in the interior becomes large. This way massive field theories are mapped to nearly AdS spaces.

The radial direction in AdS emerges without being a space dimension in 
the field theory. It can be interpreted as the renormalization group scale, or the energy scale used to probe the theory. The asymptotic region corresponds to the UV region of the field theory. This is where the theory is formulated, and this is where the operators are defined. The interior of the space corresponds to the IR region of the field theory. It is determined from the definition of the theory in the UV.

A crucial fact which underlies the correspondence, is the infinite warp factor at the boundary of the AdS space. Because of this warp factor, finite distances in the field theory correspond to infinite distances in the bulk. Therefore, a field theory correlation function of finitely separated operators is mapped to a gravity problem which infinitely separated sources.

An important consequence of this infinite warp factor is the effect of finite temperature. The boundary field theory can be put at finite temperature $T$ by compactifying its Euclidean time direction on a finite circle of radius $R=\frac{1}{2 \pi T}$. At low temperature, the only change in the dual asymptotically AdS background it to compactify its Euclidean time. Because of the infinite warp factor, the radius of the Euclidean time circle in the AdS space is large near the boundary, and it is small only in a region of the size of the AdS radius $R_{A d S}$. Therefore, most of the bulk of the space is cold. Only a finite region in the interior is hot. As the system is heated up, the boundary theory undergoes a thermal deconfinement phase transition. In the bulk it is mapped to the appearance of a Schwarzschild horizon at small radius and the topology is such that the Euclidean time circle becomes contractible. For a CFT on a 3 -sphere, this phase transition is the Hawking-Page transition, and the dual high temperature background is AdS-Schwarzschild. Both above and below the transition the bulk asymptotes to (nearly) AdS. Most of it remains cold and it is not sensitive to the short distance behavior of string theory.

While the boundary field theory is manifestly local, locality in the bulk is subtle. Because of the infinite warp factor, possible violation of locality in the bulk over distances of order $l_{s}$ could be consistent with locality at the 
boundary. In fact, it is quite difficult to find operators in the field theory which represent events in the bulk which are localized on scales of order $R_{A d S}$ or smaller. This underscores the fact that it is not clear what we mean by locality, if all we can measure are observables at infinity.

These developments have led to many new insights about the two sides of the duality and the relation between them (for a review, see [21]). In particular, many new results about gauge theories, including their strong coupling phenomena like thermal phase transitions, confinement and chiral symmetry breaking were elucidated. The main new insight about gravity is its holographic nature - the boundary theory contains all the information about the bulk gravity theory which is higher dimensional. Therefore, the number of degrees of freedom of a gravity theory is not extensive. This is consistent with the lack of local observables in gravity.

\subsection{Emergent space with gravity: linear dilaton back- grounds}

\subsubsection{Generalities}

Another class of examples of an emergent space dimension involves backgrounds with a linear dilaton direction. The string coupling constant depends on the position in the emergent direction, parameterized by the spatial coordinate $\phi$, through $g_{s}(\phi)=e^{\frac{Q \phi}{2}}$ with an appropriate constant $Q$. Therefore, the string coupling constant vanishes at the boundary $\phi \rightarrow-\infty$. The other end of the space at $\phi \rightarrow+\infty$ is effectively compact.

Like the AdS examples, here the bulk string theory is also dual to a theory without gravity at the boundary. In that sense, this is another example of holography. However, there are a few important differences between this duality and the AdS/CFT duality.

In most of the linear dilaton examples the holographic theory is not a standard local quantum field theory. For example, the near horizon geometry of a stack of NS5-branes is a linear dilaton background which is holographic 
to the little string theory (for a review, see e.g. [11]). The stringy, non-field theoretic nature of the holographic theory follows from the fact that it has nonzero $\alpha^{\prime}$, and therefore it exhibits T-duality.

Because of the vanishing interactions at the boundary of the space, the interactions take place in an effectively compact region (the strong coupling end). Therefore, we can study the S-matrix elements of the bulk theory. These are the observables of the boundary theory.

Unlike the AdS examples, the string metric does not have an infinite warp factor. Here finite distances in the boundary theory correspond to finite distances (in string units) in the bulk. Therefore, it is difficult to define local observables in the boundary theory and as a result, the holographic theory is not a local quantum field theory.

This lack of the infinite warp factor affects also the finite temperature behavior of the system. Finite temperature in the boundary theory is dual to finite temperature in the entire bulk. Hence, the holographic theory can exhibit Hagedorn behavior and have maximal temperature.

\subsubsection{Matrix model duals of linear dilaton backgrounds}

Even though the generic linear dilaton theory is dual to a complicated boundary theory, there are a few simple cases where the holographic theories are very simple and are given by the large $N$ limit of certain matrix models.

The simplest cases involve strings in one dimension $\phi$ with a linear dilaton. The string worldsheet theory includes a Liouville field $\phi$ and a $c<1$ minimal model (or in the type 0 theory a $\hat{c}<1$ superminimal model). The holographic description of these minimal string theories is in terms of the large $N$ limit of matrix integrals (for a review, see e.g. [22]).

Richer theories involve strings in two dimensions: a linear dilaton direction $\phi$ and time $x$ (for a review, see e.g. [23]). Here the holographic theory is the large $N$ limit of matrix quantum mechanics.

These two-dimensional string theories have a finite number of particle 
species. The bosonic string and the supersymmetric $0 \mathrm{~A}$ theory have one massless boson, and the $\mathrm{OB}$ theory has two massless bosons. Therefore, these theories do not have the familiar Hagedorn density of states of higher dimensional string theories, and correspondingly, their finite temperature behavior is smooth.

One can view the finite temperature system as a system with compact Euclidean time $x$. Then, the system has $R \rightarrow \alpha^{\prime} / R$ T-duality which relates high and low temperature. As a check, the smooth answers for the thermodynamical quantities respect this T-duality.

It is important to distinguish the two different ways matrix models lead to emergent space. Above (section 5.2) we discussed the emergence of the two-dimensional string worldsheet with its worldsheet general covariance. Here, we discuss the target space of this string theory with the emergent holographic dimension $\phi$.

Since the emergence of the holographic direction in these systems is very explicit, we can use them to address various questions about this direction. In particular, it seems that there are a number of inequivalent ways to describe this dimension. The most obvious description is in terms of the Liouville field $\phi$. A second possibility is to use a free worldsheet field which is related to $\phi$ through a nonlocal transformation (similar to T-duality transformation). This is the Backlund field of Liouville theory. A third possibility, which is also related to $\phi$ in a nonlocal way arises more naturally out of the matrices as their eigenvalue direction. These different descriptions of the emergent direction demonstrate again that the ambiguity in the description of space which we reviewed above (section 2) is not limited to compact dimensions. It also highlights the question of locality in the space. In which of these descriptions do we expect the theory to be local? Do we expect locality in one of them, or in all of them, or perhaps in none of them? 


\subsubsection{2d heterotic strings}

We would like to end this subsection with a short discussion of the heterotic two-dimensional linear dilaton system. Even though there is no known holographic matrix model dual of this system, some of its peculiar properties can be analyzed.

As with the two-dimensional linear dilaton bosonic and type 0 theories, this theory also has a finite number of massless particles. But here the thermodynamics is more subtle. We again compactify Euclidean time on a circle of radius $R$. The worldsheet analysis shows that the system has $R \rightarrow \alpha^{\prime} / 2 R$ T-duality. Indeed, the string amplitudes respect this symmetry. However, unlike the simpler bosonic system, here the answers are not smooth at the selfdual point $R=\sqrt{\alpha^{\prime} / 2}$. This lack of smoothness is related to long macroscopic strings excitations [24].

What is puzzling about these results is that they cannot be interpreted as standard thermodynamics. If we try to interpret the Euclidean time circle as a thermal ensemble with temperature $T=\frac{1}{2 \pi R}$, then the transition at $R=$ $\sqrt{\alpha^{\prime} / 2}$ has negative latent heat. This violates standard thermodynamical inequalities which follow from the fact that the partition function can be written as a trace over a Hilbert space $\operatorname{Tr} e^{-H / T}$ for some Hamiltonian $H$. Therefore, we seem to have a contradiction between compactified Euclidean time and finite temperature. The familiar relation between them follows from the existence of a Hamiltonian which generates local time evolution. Perhaps this contradiction means that we cannot simultaneously have locality in the circle and in its T-dual circle. For large $R$ the Euclidean circle answers agree with the thermal answers with low temperature. But while these large $R$ answers can be extended to smaller $R$, the finite temperature interpretation ceases to make sense at the selfdual point. Instead, for smaller $R$ we can use the T-dual circle, which is large, and describe the T-dual system as having low temperature. 


\subsection{Emergent space in the BFSS matrix model}

As a final example of emergent space we consider the BFSS matrix model (for a review, see e.g. 25]). Its starting point is a large collection of D0-branes in the lightcone frame. The lightcone coordinate $x^{+}$is fundamental and the theory is an ordinary quantum mechanical system with $x^{+}$being the time.

The transverse coordinates of the branes $x^{i}$ are the variables in the quantum mechanical system. They are not numbers. They are $N$ dimensional matrices. The standard interpretation as positions of the branes arises only when the branes are far apart. Then the matrices are approximately diagonal and their eigenvalues are the positions of the branes. In that sense the transverse dimensions emerge from the simple quantum mechanical system.

The remaining spacetime direction, $x^{-}$, emerges holographically. It is related to the size of the matrices $N \sim p_{-}$where $p_{-}$is the momentum conjugate to $x^{-}$.

\section{Emergent time}

After motivating the emergence of space it is natural to ask whether time can also emerge. One reason to expect it is that this will put space and time on equal footing - if space emerges, so should time. This suggests that time is also not fundamental. The theory will be formulated without reference to time and an approximate (classical) notion of macroscopic time, which is our familiar "time", will emerge. Microscopically, the notion of time will be ill defined and time will be fuzzy.

There are several obvious arguments that time should not be emergent:

1. Even though we have several examples of emergent space, we do not have a single example of emergent time.

2. We have mentioned some of the issues associated with locality in emergent space. If time is also emergent we are in danger of violating locality 
in time and that might lead to violation of causality.

3. It is particularly confusing what it means to have a theory without fundamental time. Physics is about predicting the outcome of an experiment before the experiment is performed. How can this happen without fundamental time and without notions of "before and after"? Equivalently, physics is about describing the evolution of a system. How can systems evolve without an underlying time? Perhaps these questions can be avoided, if some order of events is well defined without an underlying time.

4. More technically, we can ask how much of the standard setup of quantum mechanics should be preserved. In particular, is there a wave function? What is its probabilistic interpretation? Is there a Hilbert space of all possible wave functions, or is the wave function unique? What do we mean by unitarity (we cannot have unitary evolution, because without time there is no evolution)? Some of these questions are discussed in [26].

My personal prejudice is that these objections and questions are not obstacles to emergent time. Instead, they should be viewed as challenges and perhaps even clues to the answers.

Such an understanding of time (or lack thereof) will have, among other things, immediate implications for the physics of space-like and null singularities (for a review, see e.g. [27]) like the black hole singularity and the cosmological singularity. We can speculate that understanding how time emerges and what one means by a wave function will explain the meaning of the wave-function of the Universe. Understanding this wave function, or equivalently understanding the proper initial conditions for the Universe, might help resolving some of the perplexing questions of vacuum selection in string theory. For a review of some aspects of these questions see [8]. 


\section{Conclusions and speculations}

We have argued that spacetime is likely to be an emergent concept. The fundamental formulation of the theory will not have spacetime and it will emerge as an approximate, classical concept which is valid only macroscopically.

One challenge is to have emergent spacetime, while preserving some locality - at least macroscopic locality, causality, analyticity, etc. Particularly challenging are the obstacles to formulating physics without time. It is clear that in order to resolve them many of our standard ideas about physics will have to be revolutionized. This will undoubtedly shed new light on the fundamental structure of the theory.

Understanding how time emerges will also have other implications. It will address deep issues like the cosmological singularity and the origin of the Universe.

We would like to end this talk with two general speculative comments.

Examining the known examples of a complete formulation of string theory, like the various matrix models, AdS/CFT, etc., a disturbing fact becomes clear. It seems that many different definitions lead to a consistent string theory in some background. In particular, perhaps every local quantum field theory can be used as a boundary theory to define string theory in (nearly) AdS space. Perhaps every quantum mechanical system can be the holographic description of string theory in $1+1$ dimensions. And perhaps even every ordinary integral defines string theory in one Euclidean dimension. With so many different definitions we are tempted to conclude that we should not ask the question: "What is string theory?" Instead, we should ask: "Which string theories have macroscopic dimensions?" Although we do not have an answer to this question, it seems that large $N$ will play an important role in the answer.

Our second general comment is about reductionism - the idea that science at one length scale is derived (at least in principle) from science at shorter scales. This idea has always been a theme in all branches of science. However, 
if there is a basic length scale, below which the notion of space (and time) does not make sense, we cannot derive the principles there from deeper principles at shorter distances. Therefore, once we understand how spacetime emerges, we could still look for more basic fundamental laws, but these laws will not operate at shorter distances. This follows from the simple fact that the notion of "shorter distances" will no longer make sense. This might mean the end of standard reductionism.

Acknowledgments: We would like to thank the organizers of the 23rd Solvay Conference in Physics for arranging such an interesting and stimulating meeting and for inviting me to give this talk. We also thank T. Banks, I. Klebanov, J. Maldacena, and D. Shih for useful comments and suggestions about this rapporteur talk. This research is supported in part by DOE grant DE-FG02-90ER40542.

\section{References}

[1] M. B. Green, J. H. Schwarz and E. Witten, "Superstring Theory. Vol. 1: Introduction," Cambridge, Uk: Univ. Pr. (1987), "Superstring Theory. Vol. 2: Loop Amplitudes, Anomalies And Phenomenology," Cambridge, Uk: Univ. Pr. (1987).

[2] J. Polchinski, "String theory. Vol. 1: An introduction to the bosonic string," Cambridge, UK: Univ. Pr. (1998), "String theory. Vol. 2: Superstring theory and beyond," Cambridge, UK: Univ. Pr. (1998).

[3] A. Giveon, M. Porrati and E. Rabinovici, "Target space duality in string theory," Phys. Rept. 244 (1994) 77 arXiv:hep-th/9401139.

[4] A. Sen, "An introduction to duality symmetries in string theory," Prepared for Les Houches Summer School: Session 76: Euro Summer School 
on Unity of Fundamental Physics: Gravity, Gauge Theory and Strings, Les Houches, France, 30 Jul - 31 Aug 2001

[5] K. Hori, S. Katz, A. Klemm, R. Pandharipande, R. Thomas, C. Vafa, R. Vakil and E. Zaslow, "Mirror Symmetry" Clay Mathematics Monographs Vol 1 (AMS, 2003).

[6] R. Dijkgraaf, Rapporteur talk at this conference.

[7] V. Fateev, A.B. Zamolodchikov and Al.B. Zamolodchikov, unpublished.

[8] J. Polchinski, Rapporteur talk at this conference.

[9] E. Witten, "Conformal field theory in four and six dimensions," Prepared for Symposium on Topology, Geometry and Quantum Field Theory (Segalfest), Oxford, England, United Kingdom, 24-29 Jun 2002

[10] M. R. Douglas and N. A. Nekrasov, "Noncommutative field theory," Rev. Mod. Phys. 73 (2001) 977 arXiv:hep-th/0106048].

[11] O. Aharony, "A brief review of 'little string theories'," Class. Quant. Grav. 17 (2000) 929 arXiv:hep-th/9911147|.

[12] N. Seiberg, "The power of duality: Exact results in 4D SUSY field theory," Int. J. Mod. Phys. A 16 (2001) 4365 arXiv:hep-th/9506077.

[13] K. A. Intriligator and N. Seiberg, "Lectures on supersymmetric gauge theories and electric-magnetic duality," Nucl. Phys. Proc. Suppl. 45BC (1996) 1 arXiv:hep-th/9509066.

[14] S. Weinberg and E. Witten, "Limits On Massless Particles," Phys. Lett. B 96 (1980) 59.

[15] Y. Makeenko, "Methods of contemporary gauge theory," Cambridge, UK: Univ. Pr. (2002). 
[16] R. C. Myers, "Dielectric-branes," JHEP $9912 \quad$ (1999) 022 arXiv:hep-th/9910053.

[17] P. H. Ginsparg and G. W. Moore, "Lectures on 2-D gravity and 2-D string theory," arXiv:hep-th/9304011.

[18] J. M. Maldacena, "The large N limit of superconformal field theories and supergravity," Adv. Theor. Math. Phys. 2 (1998) 231 [Int. J. Theor. Phys. 38 (1999) 1113] arXiv:hep-th/9711200|.

[19] S. S. Gubser, I. R. Klebanov and A. M. Polyakov, "Gauge theory correlators from non-critical string theory," Phys. Lett. B 428 (1998) 105 arXiv:hep-th/9802109.

[20] E. Witten, "Anti-de Sitter space and holography," Adv. Theor. Math. Phys. 2 (1998) 253 arXiv:hep-th/9802150.

[21] O. Aharony, S. S. Gubser, J. M. Maldacena, H. Ooguri and Y. Oz, "Large N field theories, string theory and gravity," Phys. Rept. 323 (2000) 183 arXiv:hep-th/9905111.

[22] N. Seiberg and D. Shih, "Minimal string theory," Comptes Rendus Physique 6 (2005) 165 arXiv:hep-th/0409306.

[23] I. R. Klebanov, "String theory in two-dimensions," arXiv:hep-th/9108019.

[24] N. Seiberg, "Long strings, anomaly cancellation, phase transitions, Tduality and locality in the 2d heterotic string," arXiv:hep-th/0511220.

[25] T. Banks, "TASI lectures on matrix theory," arXiv:hep-th/9911068.

[26] J. Hartle, Rapporteur talk at this conference.

[27] G. Gibbons, Rapporteur talk at this conference. 